\title{
Correction to: Comprehensive Registry of Esophageal Cancer in Japan, 2011
}

\author{
Yuji Tachimori $^{1}$ (D) Soji Ozawa ${ }^{2} \cdot$ Hodaka Numasaki $^{3} \cdot$ Ryu Ishihara $^{4} \cdot$ Hisahiro Matsubara $^{5} \cdot$ Kei Muro $^{6}$. \\ Tsuneo Oyama ${ }^{7}$. Yasushi Toh $^{8} \cdot$ Harushi Udagawa $^{9} \cdot$ Takashi Uno $^{10} \cdot$ The Registration Committee for Esophageal \\ Cancer of the Japan Esophageal Society
}

Published online: 12 June 2018

(c) The Japan Esophageal Society and Springer Japan KK, part of Springer Nature 2018

\section{Correction to: Esophagus https://doi.org/10.1007/s10388-018-0614-z}

In the original publication of the article, the below name of institutions were not included in the table of Institutionregistered cases in 2011.

\section{List of institutions}

Keio University Hospital

Keiyukai Sapporo Hospital

Kikuna Memorial Hospital

Kinki Central Hospital

Kinki University Hospital

Kiryu Kosei General Hospital

Kishiwada City Hospital

Kitaakita Municipal Hospital

Kitakyushu Municipal Medical Center

Kitano Hospital

Kitasato Institute Hospital

The original article can be found online at https://doi.org/10.1007/ s10388-018-0614-z.

Yuji Tachimori

y-tachimori@saiwaihp.jp

1 Cancer Care Center, Kawasaki Saiwai Hospital, 31-27 Omiya-cho, Saiwai-ku, Kawasaki, Kanagawa 212-0014, Japan

2 Department of Gastroenterological Surgery, Tokai University School of Medicine, Isehara, Japan

3 Department of Medical Physics and Engineering, Osaka University Graduate School of Medicine, Osaka, Japan

4 Department of Gastrointestinal Oncology, Osaka International Cancer Institute, Osaka, Japan

5 Department of Frontier Surgery, Graduate School of Medicine, Chiba University, Chiba, Japan
Kitasato University Hospital

Kobe City Medical Center General Hospital

Kobe University Hospital

Kochi Health Sciences Center

Kochi University Hospital

Kokura Memorial Hospital

Kumagaya General Hospital

Kumamoto City Hospital

Kumamoto University Hospital

Kurashiki Central Hospital

Kurume General Hospital

Kurume University Hospital

Kyoto University Hospital

Kyushu Central Hospital of the Mutual Aid Association of Public School Teachers

Kyushu Medical Center

Kyushu University Beppu Hospital

Kyushu University Hospital

Machida Municipal Hospital

Matsuda Hospital

Matsudo City Hospital

Matsushita Memorial Hospital

6 Department of Clinical Oncology, Aichi Cancer Center Hospital, Nagoya, Japan

7 Department of Gastroenterology, Saku General Hospital, Nagano, Japan

8 Department of Gastroenterological Surgery, National Kyushu Cancer Center, Fukuoka, Japan

9 Department of Gastroenterological Surgery, Toranomon Hospital, Tokyo, Japan

10 Department of Radiology, Graduate School of Medicine, Chiba University, Chiba, Japan 
Matsuyama Red Cross Hospital

Mie University Hospital

Minamiosaka Hospital

Mino City Hospital

Mito Red Cross Hospital

Mitsui Memorial Hospital

Miyazaki Konan Hospital

Murakami General Hospital

Musashino Red Cross Hospital

Nagahama City Hospital
Nagano Red Cross Hospital

Nagaoka Chuo General Hospital

Nagasaki University Hospital

Nagoya City East Medical Center

Nagoya City University Hospital

Nagoya Daiichi Red Cross Hospital

Nagoya University Hospital

Nanpuh Hospital 